\title{
IMPLICACIONES TRANSNACIONALES EN LA HISTORIA DE LA PSIQUIATRÍA: LA REUBICACIÓN DE PACIENTES DE TIROL DEL SUR (ITALIA) EN INSTITUCIONES PSIQUIÁTRICAS ALEMANAS, 1940-1945
}

\author{
Thomas Müller \\ Priv.-Doz. Dr. med. Thomas Müller, M.A. \\ th.mueller@zfp-zentrum.de \\ Uta Kanis-Seyfried \\ Dr. Uta Kanis-Seyfried, M.A. \\ uta.kanis-seyfried@zfp-zentrum.de
}

Recibido: 8 febrero 2016; Aceptado: 29 abril 2016

\begin{abstract}
Cómo citar este artículo/Citation: Müller, Thomas y Kanis-Seyfried, Uta (2016), "Implicaciones transnacionales en la historia de la psiquiatría: la reubicación de pacientes de Tirol del sur (Italia) en instituciones psiquiátricas alemanas, 1940-1945", Asclepio, 68 (2): p160. doi: http://dx.doi.org/10.3989/asclepio.2016.32

RESUMEN: El objeto de este trabajo es investigar el destino de un grupo de pacientes psiquiátricos ingresados en asilos de Württemberg (Alemania) durante el periodo de 1940 a 1945. Dichos internos, procedentes principalmente de las regiones italianas de Vicenza, Udine, Trento y Alto Adigio, fueron enviados (y un número importante entre ellos ilegalmente deportados) a los psiquátricos alemanes de Zwiefalten, Schussenried y Weissenau, situados todos en el sur de la región alemana de Württemberg, entre 1940 y 1943. Especial atención se dedica a los llamados Acuerdos para la Opción entre el Reich alemán y la Italia fascista, como parte de la biopolítica nacionalsocialista. El tratamiento de estos pacientes surtiroleses en los asilos, así como su destino, se sitúa en el contexto de las acciones de reubicación de la población realizadas en las zonas limítrofes del Tercer Reich, que comenzaron en 1939 y afectaron a amplias zonas del continente europeo. Se estudian comparativamente también en esta contribución otros subgrupos poblacionales que emigraron por entonces de Italia al Reich alemán.
\end{abstract}

PALABRAS CLAVE: Psiquiatría; Alemania nazi; Italia fascista; Acuerdos para la Opción; Eutanasia descentralizada.

\section{TRANSNATIONAL ENTANGLEMENTS IN THE HISTORY OF PSYCHIATRY. SOUTH TYROLIAN PATIENTS IN GERMAN ASYLUMS, C. 1940-1945}

\begin{abstract}
Object of this article is the destiny of psychiatric inmates in Wuerttemberg asylums, 1940-1945. These patients from Italian regions of Vicenza, Udine, Trento, Alto Adige and various locations more were delegated and, to a substantial number, illegally deported to the German asylums Zwiefalten, Schussenried and Weissenau, all in South Wuerttemberg, in 1940 and 1943. Attention is focused on the pioneering state pre-negotiations, and the so-called option treaties between the German Reich and Fascist Italy as part of the general aspect of National Socialist bio-Politics. The treatment of these South Tyrol patients in the asylums themselves, as well as their fate will be put into the context of the resettlement actions at the margins of the Third Reich, which started in 1939 and widely affected the European continent. It is referred to other sub-groups of migrating population from Italy to the German Reich as well, as a contrasting aspect of this contribution.
\end{abstract}

KEY WORDS: Psychiatry; National Socialist Germany; Fascist Italy; Option treaties; Decentralised euthanasia. 


\section{INTRODUCCIÓN}

En el contexto de la denominada «opción» entre el Tercer Reich alemán y la Italia fascista, pacientes procedentes de diferentes centros psiquiátricos del Tirol del Sur, el Trentino y otras provincias italianas colindantes fueron trasladados a partir de 1939 a Alemania (entre ellos, algunos de forma no voluntaria), pasando ocasionalmente por el centro psiquiátrico de Hall, en el Tirol septentrional (Austria). Hall funcionó así como una especie de nudo logístico para el posterior envío de estos pacientes a los centros psiquiátricos de Zwiefalten y Schussenried, y desde este último a su vez a Ravensburg-Weissenau (los tres situados en Württemberg, Alemania).

Los pacientes que llegaron entre 1940 y 1943 a Württemberg en el marco del Acuerdo para la Opción no fueron - al menos según el estado actual de nuestras investigaciones - víctimas de la eutanasia centralizada (la realizada dentro de la Aktion T4, el programa nazi para el asesinato sistemático de enfermos psíquicos o discapacitados). Pero otros enfermos del psiquiátrico de Hall sí habrían sido trasladados directamente a Hartheim (cerca de Linz, Austria), uno de los centros de exterminio de la eutanasia centralizada, y asesinados allí. No obstante, la suerte corrida por los pacientes deportados a Württemberg dentro de la «opción» tampoco fue mucho mejor. Al final de la guerra, en 1945, casi la mitad de estas 493 personas habían fallecido. Indagar, por tanto, el destino de estos pacientes no es sólo un motivo de investigación histórico-médica, también es un imperativo moral dirigido a honrar su memoria.

Junto a los informes anuales de las instituciones psiquiátricas aludidas, utilizaremos como fuentes principales en nuestro estudio las historias clínicas de estos pacientes y también, ocasionalmente, la correspondencia con sus familiares así como diversa documentación italiana.

\section{LAS NEGOCIACIONES POLÍTICAS ENTRE LA ALEMA- NIA NAZI Y LA ITALIA FASCISTA}

El traslado de surtiroleses a Württemberg tuvo como telón de fondo las acciones de reubicación que el Tercer Reich inició en toda Europa en 1939. En mayo de ese año, el comandante en jefe de las SS, Heinrich Himmler, presentó un memorándum para la solución definitiva de la cuestión del Tirol del Sur. En él, y de acuerdo con los deseos de Adolf Hitler, el Reich renunciaba a este territorio y exigía la reubicación en territorio alemán de todos los alemanes étnicos (Volksdeutsche) allí residentes.
El memorándum supuso un duro golpe para los surtiroleses de lengua alemana o germanófilos que habían contado con una anexión del Tirol del Sur al Reich alemán. El 23 de junio de 1939 este memorándum adquirió carácter oficial con el Acuerdo de Berlín (Berliner Vereinbarung), cuyo objetivo era conseguir una "solución étnica radical para la cuestión del Alto Adigio». Según dicho acuerdo, todos los alemanes ciudadanos del Reich (Reichsdeutsche) residentes en Tirol del Sur debían emigrar al Reich alemán. A los alemanes étnicos que poseían la ciudadanía italiana se les concedía el derecho de "opción»: debían decidirse bien por la ciudadanía italiana o bien por la alemana. Esta decisión, supuestamente libre y no sujeta a presiones, se vio a menudo desvirtuada y convertida en un plebiscito en favor o en contra de Alemania, tanto por las autoridades alemanas como por una agrupación local denominada Círculo de Lucha Étnico Surtirolés (Völkischer Kampfring Südtirol, VKS) (Stuhlpfarrer, 1985, pp. 177-205). En un principio, este Círculo se pronunció incluso en contra de la "opción» (y a favor de una anexión directa del Tirol del Sur al Reich alemán), aunque luego viró hacia las posiciones dictadas por el régimen nacionalsocialista.

Aquellos que optaron por su reubicación en Alemania fueron denominados optantes (Optanten) y los que decidieron permanecer en Italia (cuyo porvenir no se auguraba muy boyante en un estado progresivamente italianizado), fueron denominados permanecientes (Dableiber). En septiembre de 1940, antes incluso de la publicación de las directrices relativas a la reubicación de la población, se instauró en Bolzano el Amtliche Deutsche Ein- und Rückwandererstelle (ADERSt) u Organismo Oficial para Inmigrantes y Repatriados Alemanes, que debía organizar in situ la reubicación con el apoyo del Arbeitsgemeinschaft der Optanten für Deutschland (AdO) o Grupo de Trabajo de los Optantes por Alemania. Ambas instancias quedaron subordinadas al también creado por entonces Dienststelle des Reichskommissars für die Festigung deutschen Volkstums (RKF) o Negociado del Comisario del Reich para la Consolidación del Carácter Nacional Alemán. A este Negociado le competía planear, organizar y coordinar todas las acciones relacionadas con la reubicación. La naturalización y el asentamiento de los tiroleses del sur fueron tareas fundamentalmente de otra instancia: el Dienststelle Umsiedlung Südtirol (DUS) o Negociado para la Reubicación del Tirol del Sur, con sede en Innsbruck.

Derecho a la «opción» tenían, según el acuerdo, todos los alemanes étnicos, así como los cimbrios, una 
minoría lingüística bávara del Véneto y del TrentinoTirol del Sur cuya lengua había prohibido Benito Mussolini en 1922, tanto en su utilización pública como privada, y que residían en las provincias de Bolzano, Trento, Belluno, Vicenza, Verona y Udine. Por exigencia expresa italiana se incluyó también entre los optantes a los ladinos: los provenientes de las comarcas de habla romanche, como Val Gardena, Val Badia, Cortina d'Ampezzo, Livinalolongo o Colle Santa Lucia, aunque no de Val di Fassa.

La única condición que debía cumplir el optante era considerase a sí mismo perteneciente al pueblo alemán. Para los alemanes étnicos menores de edad, la decisión correspondía a los padres. Pero en las directrices de la reubicación no se concretaba claramente la potestad de los familiares sobre la opción de los considerados jurídicamente incapacitados y, en especial, de los internos de las clínicas y sanatorios del Tirol del Sur. En un pasaje de dichas directrices se refiere que a los «ancianos y enfermos alemanes ciudadanos del Reich» les era permitida la permanencia en Tirol del Sur. Pero Himmler ya había dado a entender durante las negociaciones previas que Alemania estaba dispuesta a acoger a los alemanes étnicos tanto «mental y físicamente inferiores» como a los reclusos de las prisiones (Fiebrandt, 2014, pp. 562-568). La acción de traslado - a la que se calificó de "éxodo»- estaba planeada en principio para finalizarse el 31 de diciembre de 1942, pero el periodo de ejecución se acortó posteriormente, adelantándose la fecha límite a la Nochevieja de 1939, lo que resultó ser un objetivo poco realista.

La "opción» se saldó con una clara mayoría a favor del Reich: en las provincias de Bolzano y Udine, por ejemplo, más del $80 \%$ de los surtiroleses con derecho de opción se decantaron por la reubicación en el Reich. En total fueron unas 200.000 personas en todas las provincias italianas implicadas las que manifestaron su intención de emigrar.

Según el plan previsto, los emigrantes debían abandonar el Tirol del Sur en tandas sucesivas: primero los alemanes ciudadanos del Reich, a continuación la población no ligada a la tierra (nicht bodengebunden) y, finalmente, la población ligada a la tierra (bodengebunden) (por «ligada a la tierra» hay que entender fundamentalmente a la población agricultora) (Fiebrandt, 2014, pp. 135-149). Hasta la anexión por parte de Alemania de los territorios pre-alpinos del norte de Italia en septiembre de 1943 -es decir, hasta el fin de la alianza armada entre Italia y Alemania - sólo emigraron en realidad 75.000 tiroleses del sur, cimbrios y ladinos de un total de entre 200.000 a 250.000 optantes por Alemania. El objetivo primordial, y a la vez el problema fundamental que impidió que el número de emigrantes fuera mayor, fue el asentamiento en bloque de los surtiroleses. Y es que, a pesar de que los diversos planes de asentamiento ofrecían supuestas zonas libres para dicho asentamiento (áreas de Galitzia, granjas en el Warthegau, incluso áreas en la Borgoña francesa en donde se planeaba el establecimiento de granjas modélicas de las SS, o incluso la península de Crimea), tales zonas no estaban aún disponibles de facto (Wedekind, 2009).

La parte alemana intentó retrasar el transporte de los surtiroleses ligados a la tierra hasta que fuese posible su reasentamiento en zonas adecuadas. Y, dado que el Reich se había comprometido a mantener una cuota migratoria de 200 personas diarias, se reubicó en primer lugar los tiroleses del sur no ligados a la tierra, una fuerza de trabajo que podía emplearse en la economía, en la administración o en las fuerzas armadas (Helmut, 1993).

Por otro lado, a la parte italiana le interesaba que emigraran las personas que, política o financieramente, suponían una carga para el estado italiano, lo que implicaba el traslado al Reich de los enfermos psíquicos y de los ancianos alojados respectivamente en los psiquiátricos y asilos del Tirol del Sur (Fiebrandt; Rüdenburg y Müller, 2012a. También: Müller; Kanis-Seyfried, 2013). Ante las crecientes dificultades para cumplir con las cuotas migratorias previstas, dicha propuesta acabó aceptándose por las autoridades alemanas responsables de la reubicación, y además se hizo extensiva también a las prostitutas (Fiebrandt, 2014, p. 563).

La reubicación de los enfermos psíquicos se realizó inicialmente con un tren directo con casi 300 personas, el cual partió del sanatorio surtirolés de Pergine ${ }^{1}$, cerca de Trento, con destino al sanatorio de Zwiefalten, junto a Riedlingen, en Württemberg, en mayo de 1940. Entre 1940 y 1942 se efectuaron además varios transportes de menor entidad con destino a Schussenried, también en Württemberg (Fiebrandt; Rüdenburg y Müller, 2012a, pp. 171-187). Estos transportes menores se llevaron a cabo pasando por el sanatorio tirolés de Hall, objeto de investigación en la actualidad ${ }^{2}$, y que acabó convirtiéndose en una especie de estación de recogida para los enfermos psíquicos procedentes del norte de Italia. Schussenried fue incluido en los planes porque, tras la llegada del primer transporte, la capacidad de Zwiefalten -incluso con la rebaja de los estándares de los tiempos 
de guerra - se consideró totalmente agotada. Cuando la ocupación de Schussenried alcanzó también un límite máximo, algunos pacientes allí internados fueron trasladados de nuevo, esta vez al antiguo centro de Weissenau, junto a Ravensburg.

\section{PACIENTES PSIQUIÁTRICOS SURTIROLESES PROCE- DENTES DE PERGINE E INGRESADOS EN ZWIEFALTEN}

Nos ocuparemos ahora con mayor detalle del traslado de estos 299 pacientes de Pergine a Zwiefalten. El punto de partida de las negociaciones sobre el traslado de pacientes de centros psiquiátricos del Tirol del Sur al Reich alemán fue presumiblemente una conversación mantenida en abril de 1940 en Bolzano. En ella el prefecto de dicha ciudad propuso al jefe de la oficina principal del RKF un primer transporte de sólo 40 enfermos mentales, si bien era un total de unos 600 los que habrían de ser trasladados desde su prefectura (Fiebrandt; Rüdenburg y Müller, 2012a, p. 165; y también Arreghini, 1995). Poco tiempo después, el prefecto solicitó información en el ADERSt de Bolzano acerca de si se habían recibido instrucciones de Berlín sobre el destino del transporte ${ }^{3}$. Esto indica, de un lado, que en ese momento era la parte italiana la más interesada en una rápida deportación de los enfermos; y de otro, que por parte alemana quedaban todavía aspectos organizativos pendientes de clarificación, que sólo quedarían solucionados en mayo de 1940. Los paralelismos con respecto al modo de proceder en la reubicación de los alemanes del Báltico (Baltendeutsche) son evidentes (Fiebrandt, 2014, pp. 123-136 y 557-613), pues ya en diciembre de 1939 y en enero de 1940 dos grandes transportes con alemanes del Báltico afectos de enfermedades psíquicas habían abandonado Letonia en dirección al Reich.

Pero volvamos a nuestro contexto. En Bolzano se acordó además trasladar a todos los pacientes primero al psiquiátrico de Pergine, al objeto de transferirlos desde allí en bloque a Württemberg. El éxodo debía efectuarse por medio de un pasaporte colectivo. Además, el director del sanatorio de Zwiefalten asumiría provisionalmente la tutela de todos los pacientes, cuya opción voluntaria a favor de Alemania debería haber sido un requisito necesario para su reubicación, aunque en realidad ésta sólo constaba en un escaso número de casos. El informe sobre el transporte hace únicamente la siguiente observación sobre la incapacidad jurídica y, con ello, sobre la aceptación de la opción de estos surtiroleses: «Naturalmente, los enfermos no estaban en condiciones de emitir por sí mismos su voto a favor de Alemania; con algunos de ellos, la decisión fue tomada por sus parientes; en el resto de casos, la pertenencia al pueblo alemán pudo probarse por el lugar de nacimiento y el apellido» ${ }^{4}$. Tras este paso, sus pasaportes italianos dejaron de tener validez.

De iure, tal procedimiento sumario no era admisible ni siquiera con la legislación entonces vigente. A esto se sumaba que, según el derecho italiano de la época, la admisión en un centro psiquiátrico conllevaba la pérdida de los derechos civiles, incluido el derecho a voto, por lo que los pacientes deberían haber quedado excluidos, incluso pro forma, de la opción ${ }^{5}$. Pero como no se presentaron objeciones por ninguna de las partes, en mayo de 1940 comenzaron los preparativos para los transportes. En Pergine tuvieron lugar, en presencia del director del centro, el doctor Alberto Rezza, las conversaciones finales. Junto al prefecto de Bolzano, una participación determinante en la organización y ejecución del transporte tuvieron también el doctor Walther Simek, encargado del Departamento de Exteriores de la Reichsärztekammer (RÄK) o Cámara Médica del Reich y los doctores Reinhold Wetjen y Hermann Pedoth, empleados del ADERSt de Bolzano. El personal de acompañamiento a los enfermos lo componían enfermeras y enfermeros del centro de Pergine.

El DUS de Innsbruck no desempeñó papel alguno en la preparación y ejecución del transporte, por lo que es posible que en ningún momento se tuviera intención de naturalizar a los pacientes surtiroleses. El traslado se produjo finalmente el 26 de mayo de 1940, sólo dos días después de que hubieran llegado a Pergine los últimos pacientes procedentes de los centros psiquiátricos de Stadlhof, Gemona, Nomi y Udine. El convoy especial para los finalmente 299 pacientes de Pergine fue dispuesto por parte italiana. A su llegada a Zwiefaltendorf, en Württemberg, el convoy fue recibido por el director del centro de Zwiefalten, el doctor Alfons Stegmann, y por - literalmente- el «número necesario de guardianes y guardianas de enfermos». El «desalojo de los vagones» de los pacientes, transcurrió no obstante con muchos problemas. Según el informe de Stegmann, «la descarga y el transporte de los enfermos presentó notables dificultades, ya que la mayoría de ellos está débil y algunos en un estado de agitación excesiva». Además, continuaba el informe, los sujetos presentaban casi «sin excepción una carencia total de disciplina e higiene ${ }^{6}$.

Según otro informe, éste del consejero médico Alois Bischoff, quien en 1940 dirigió un cierto tiempo el sanatorio de Weissenau, al cual fueron trasladados 75 
pacientes de los llegados a Schussenried (la distancia entre ambos centros es de unos 30 kilómetros), la totalidad de estos 75 enfermos se encontraba en mal estado de salud ${ }^{7}$. A todo ello se sumó que el entendimiento con los enfermos resultó complicado, pues no todos los pacientes surtiroleses dominaban la lengua alemana. Además las historias clínicas, así como sus extractos, llegaron exclusivamente en lengua italiana. Debido a lo cual, relata Stegmann, las semanas siguientes hubieron de emplearse principalmente en traducirlas al alemán con ayuda de un paciente que hablaba ambos idiomas ${ }^{8}$.

Todo parece indicar que lo más importante para ambas partes era que el traslado de los pacientes, completado también desde el punto de vista administrativo con la expedición del certificado de expatriación, se hubiese llevado a cabo, y no tanto la suerte de los afectados. Esta percepción parece confirmarse por el hecho de que, acabado el transporte, se organizase un viaje turístico a Stuttgart y Múnich para el personal médico acompañante, como colofón a esta cooperación germano-italiana.

Consecuentemente, en su informe final sobre esta acción, Stegmann destacó especialmente que el personal italiano había sido extremadamente solícito y que había dejado "grandes cantidades de frutos meridionales, chocolate y otros comestibles para su posterior utilización». Asimismo, los colegas italianos habrían expresado «su reconocimiento expreso por la calidad del centro y su limpieza»".

Una impresión totalmente distinta de la situación y del lugar tuvo sin embargo la doctora Helene Volk, quien trabajó a finales de la década de 1930 como médica voluntaria en Zwiefalten, y que en verano de 1940 hizo una visita casual a su anterior lugar de trabajo. En su declaración en el "Proceso Grafeneck» (Kinzig y Stöckle, 2011; y también Müller; Kinzig y Stöckle, 2010), en donde los responsables de la Aktion T4 rindieron cuentas en cierta medida acabada la guerra, hizo constar en acta lo siguiente: «El largo pasillo del otrora monasterio estaba repleto de pacientes. Yacían en el suelo, en sillas, en sacos de paja, sobre las mesas, totalmente revueltos, mayores y jóvenes, personas informes, deformes, totalmente rapadas, a las que se les había escrito con tinta azul un número en la frente y en el antebrazo. También las salas de enfermos estaban repletas de estos desgraciados 'números'. [...] Nunca me he vuelto a enfrentar con una acumulación tal de miseria humana» ${ }^{10}$.

A diferencia de los responsables de Zwiefalten, la dirección del sanatorio vecino de Schussenried no se mostró tan satisfecha con el desarrollo de la reubicación. En una carta del 5 de noviembre de 1940 dirigida al Ministerio del Interior de Württemberg se lamentaba «que los enfermos incapacitados para caminar debieron ser transportados uno a uno durante un buen trecho hasta llegar a los distintos departamentos, a la vez que la oscuridad total y la lluvia persistente dificultaron sumamente el trabajo». El personal acompañante italiano no aportó ayuda alguna, pues se mostró "reiteradamente pasivo ${ }^{11}$. Además, los pacientes habían «llegado en un estado de gran abandono, sucios y llenos de piojos, tanto de la cabeza como del cuerpo». También provocaron disgusto los pacientes calificados como "enfermizos», ya que eran muy "exigentes con los cuidados» y reclamaban «a menudo medicamentos innecesarios, sobre todo tranquilizantes». Los enfermos habrían rechazado colaborar en los trabajos del centro alegando que «no estaban obligados a ello», aunque "algunos habrían estado ciertamente en condiciones de hacerlo». A los médicos nacionalsocialistas les pareció que «los pacientes estaban un poco mimados ${ }^{12}$.

La actitud crítica de estos pacientes - al menos de los que eran capaces de defenderse- queda patente en una carta de queja que algunos surtiroleses alojados en Schussenried dirigieron a la «Encomiable Dirección para Emigrantes y Repatriados». En ella dejaron constancia de su enfado por la mala comida y por el escaso tabaco recibido, así como por la ausencia de paga: «En Innsbruck se halla escrito que la Gran Alemania da la bienvenida a los tiroleses del sur. Nosotros creemos que, si nuestro querido Führer supiera el trato que en realidad se nos da, se acabarían nuestras penurias. $Y$ a este trato le llaman encima comunidad nacional $»^{13}$.

En un escrito dirigido a la Cámara Médica del Reich de Berlín, el director del Departamento de Sanidad del Ministerio del Interior de Württemberg, el consejero médico Eugen Stähle, informaba sobre la inspección efectuada posteriormente en el sanatorio de Schussenried. Según sus palabras, a los pacientes ya se les estaba dando una paga para sus gastos menores $y$, en referencia a la comida, él mismo se había convencido personalmente, "a través de una prueba de cata", de su «buena calidad». La protesta - decía- era del todo «inconsistente ${ }^{14}$, «todo el mundo debería saber que las quejas de los enfermos mentales no pueden ser tomadas en serio sin más, y que las personas mayores y enfermizas tienen mucha tendencia a quejarse continuamente». Además, según Stähle, los redactores de la carta de protesta «padecían de alcoholismo 
crónico», "esquizofrenia paranoide» y «lúes». Que a finales de julio de 1940 hubieran muerto ya 14 procedentes del Tirol del Sur lo explicaba por la elevada edad de los "pacientes enfermizos» así como por la extensión de la tuberculosis ${ }^{15}$.

Aunque no tanto como en Zwiefalten, en Schussenried y en Weissenau la tasa de mortalidad entre los pacientes surtiroleses fue también elevada ${ }^{16}$. Esta alta mortalidad podría haberse debido a diferentes causas, no sólo al estado de salud de los pacientes, sino también a la progresiva necesidad, impuesta por la guerra, de dedicar dependencias del centro a otros fines, como fue el caso del hospital militar dispuesto en Weissenau con aproximadamente 300 camas. Weissenau se convirtió además durante la guerra en lugar de alojamiento para los trabajadores forzados en la industria de la cercana Friedrichshafen, capital para la guerra. Todo ello motivó que los pacientes psiquiátricos quedaran cada vez más hacinados en el reducido espacio restante del centro (Kretschmer, 1983). Es de suponer que contribuyera también a la elevada mortalidad el deficiente suministro de medicamentos y alimentos, el cual - como puso de manifiesto para los centros de Baden Heinz Faulstich, fallecido en 2014 (Faulstich, 1998, pp. 301-305 y 351-355) - no sólo fue consecuencia de la escasez general debida a la guerra, sino que respondió también a objetivos de corte utilitarista social (Süß, 2003).

El traslado de surtiroleses con enfermedades psíquicas y discapacidades mentales desde Hall, en el Tirol austríaco, hasta Schussenried se produjo, por otro lado, en circunstancias distintas a las del traslado de los pacientes de Pergine. A Schussenried llegaron mayoritariamente sujetos que vivían con sus familiares, cuyas enfermedades no se hicieron constar en expedientes ni se consideró que precisaran terapia hasta que la reubicación ya estaba en curso, momento en el que se procedió a su psiquiatrización. Algunos pocos habían estado ya ingresados ciertamente de forma breve en algún sanatorio, pero después habían sido enviados a casa.

\section{«EUTANASIA» CENTRALIZADA O DESCENTRALIZADA. EL DESTINO DE LOS PACIENTES ITALIANOS EN ALEMANIA}

Hasta donde hoy sabemos, los casi 500 surtiroleses trasladados a Zwiefalten, Schussenried y Weissenau no fueron víctimas de la denominada eutanasia centralizada, es decir, de la Aktion T4. En Schussenried esta circunstancia se debió tan sólo a que el último transporte de la Aktion T4 a Grafeneck se realizó el 1 de noviembre de 1940, el mismo día en que los surtiroleses llegaron allí. Pero tampoco los tiroleses del sur alojados en Zwiefalten -llegados el mismo año en que desde este centro se deportaron cientos de pacientes al centro de exterminio de Grafeneck- fueron incluidos, como habría cabido en principio esperar, en la Aktion T4.

A pesar de su exclusión de la Aktion T4, los pacientes psiquiátricos surtiroleses no dejaron de tener una tasa de mortalidad Ilamativamente elevada durante el periodo bélico. En mayo de 1945 habían fallecido aproximadamente la mitad de las 299 personas trasladadas de Pergine a Zwiefalten. Esta altísima mortalidad afectó también a los pacientes «alemanes» allí residentes. ¿Por qué la mortalidad en Zwiefalten fue claramente superior a la de los centros similares de Württemberg, como Schussenried y Weissenau?

Esto se debió presumiblemente a la administración intencionada de dosis excesivas de tranquilizantes (Rüdenburg, 1996, pp. 44-46) ${ }^{17}$, proceder que, dependiendo de la forma de aplicación, puede inducir la muerte de forma lenta o rápida. Esta suposición, basada en las actuaciones de determinadas personas con responsabilidad médica, como las de la directora del centro psiquiátrico de Zwiefalten, la doctora Martha Fauser, ha podido demostrarse en algunos casos, entre otros en uno de los procesos celebrados durante la posguerra en Tübingen en 1948/49. Un problema en este sentido para nuestro trabajo es que las anotaciones de las historias clínicas disponibles sobre pacientes procedentes del Tirol del Sur o de Italia casi nunca permiten distinguir entre causas reales y falseadas de la muerte de estos pacientes, algo que sí ha podido determinarse en las historias clínicas de algunos de los asesinados dentro de la eutanasia centralizada. De este modo, puede presuponerse que los tiroleses del sur fueron incluidos en la eutanasia con medicamentos o eutanasia descentralizada, pero sin embargo no es algo -más allá de lo que ocurriera con algunos pacientes determinados- que pueda probarse todavía como algo sistemático ${ }^{18}$.

Por el contrario, la malnutrición sí que fue un motivo muy evidente de ese aumento de la mortalidad. Según los registros, el peso de no pocos pacientes se redujo drásticamente desde su llegada, llegando incluso algunos a perder la mitad, hasta no sobrepasar los 32 o 33 kilos en el momento de su muerte (Kanis-Seyfried, 2014 , p. 503). Las noticias sobre las precarias condiciones de vida del centro llegaron también a Italia, como se deduce de una carta de octubre de 1940 en la que una madre solicita, muy preocupada, información sobre el estado de su hijo, "ya que [se decía que en Zwiefalten] se han producido varias defunciones ${ }^{19}$. 
Y llegados a este punto, quizás convenga ahondar en la discusión en torno a la existencia o no de la eutanasia descentralizada, es decir, la eutanasia practicada por el personal sanitario sin que fuese ordenada por los ministerios ni por la central berlinesa responsable de la Aktion T4, a través de los ejemplos de algunos pacientes.

\section{TRASLADADOS EN "CAMISA DE FUERZA LIGERA»: ALGUNOS CASOS DE LAS «OPCIONES»}

La historia clínica de Emilia V., en la que consta un sello con la mención "reubicada surtirolesa», se encontraba en el archivo del antiguo sanatorio de Schussenried, en Württemberg ${ }^{20}$. La paciente pertenecía a los surtiroleses trasladados de Hall a Schussenried el 1 de noviembre de 1940. Pocos meses después de su Ilegada a Schussenried, el 21 de febrero de 1941, se le cumplimentó un formulario de registro en el programa de la T4 ( la "eutanasia centralizada"), que todavía se encuentra en la carpeta de su historia. Este hallazgo es esencial, ya que, por una parte, supone un indicio de la existencia de traslados previos a Grafeneck y, por otra, pone en entredicho las investigaciones de los años ochenta y noventa, en las que se negaba que los surtiroleses hubieran corrido peligro de ser víctimas de la eutanasia centralizada. En la historia clínica de Emilia V. consta que era sordomuda y que estaba diagnosticada de esquizofrenia ${ }^{21}$. En la profesión de la paciente figura «empleada de hogar». Resulta obvio que Emilia V. había caído en el radio de acción de la Aktion $T 4$, lo que la destinaba al asesinato en un centro de exterminio. Sin embargo, el centro de exterminio de Grafeneck ya había sido clausurado en ese momento. Aunque tras el cierre de Grafeneck continuaron produciéndose traslados también desde la región estudiada aquí hacia otros centros de exterminio, como por ejemplo de Weissenau a Hadamar, en Hesse, este no fue el caso de Emilia ni tampoco el de ningún otro de los pacientes de Schussenried.

Emilia V. continuó en Schussenried. Sus familiares se informaban sobre su estado con regularidad y la doctora que la trataba les respondía. En uno de estos escritos, informaba que la paciente no entendía «nada de alemán», lo cual dificultaba el tratamiento y la vida en la Unidad. Todo esto, a su vez, hace sospechar de la supuesta "opción» voluntaria de la paciente por el Reich ${ }^{22}$. En lo que respecta a su naturalización alemana, el que en 1941 fuera declarada médicamente como «incapacitada mental» pudo haber tenido consecuencias negativas. Aunque, por otro lado, en la correspondencia entre el jefe de la circunscripción territorial y el DUS del 20 de junio de 1941 aparece anotado: «No hay ningún motivo para no tratar [a la paciente] del mismo modo que al resto de surtiroleses ${ }^{23}$. Sea como fuere, la naturalización no se produjo.

Sobre su evolución médica la historia clínica nos proporciona la siguiente información: la paciente cayó enferma de un "proceso pulmonar agudo». En «su estado de debilidad y desnutrición, si no se produce pronto un cambio, [cabría] contar con un rápido fallecimiento ${ }^{24}$. En esta carpeta apenas hay datos posteriores del tratamiento médico y de la evolución de la enfermedad. Finalmente, en un escrito del 29 de junio de 1943 dirigido al DUS, junto a la noticia y la fecha de su fallecimiento, se informa también de su entierro en Schussenried, previsto para el 2 de julio de 1943 a las 13:00 horas. La cita del sepelio se puso también en conocimiento del padre de la paciente ${ }^{25}$. A la vista del apunte que consta en su expediente, similar al que se encuentra - prácticamente estandarizado- en varios expedientes de víctimas de la eutanasia descentralizada en Zwiefalten, así como ante la causa de la muerte, crece la sospecha de que nos encontramos también aquí ante una muerte inducida.

En otras historias clínicas encontramos también indicios de estas muertes inducidas. Por ejemplo, el conductor de teleféricos Franz L., nacido en Bolzano, era - según la documentación conservada - un hombre gravemente enfermo que ya durante el transporte a Württemberg había estado sujeto con una «camisa de fuerza ligera». Murió pocos días después de su llegada a Zwiefalten. Su familia, que había ejercido en su nombre el derecho de "opción», decidió volver a Italia en 1952.

Otra paciente, Katharina K., nacida en la Val Pusteria, fue calificada en su historia clínica de peligrosa, y estuvo atada durante la totalidad del viaje en tren a Alemania. Falleció en junio de 1940, también poco tiempo después de su llegada. La autopsia menciona una embolia de las arterias pulmonares. En agosto de ese mismo año todavía llegaron cartas cariñosas de su madre a Zwiefalten.

A Maria C., de la provincia de Belluno, se la describe en la historia clínica italiana de Pergine como «tranquila, hábil [...] maleable» y «no peligrosa». Pero, según la historia clínica de Zwiefalten, habría sido todo lo contrario, a saber: «muy ruidosa» y «agresiva». Padecía epilepsia y murió en junio de 1940 por una insuficiencia cardiaca y cardiovascular. En septiembre de ese año 
sus familiares, que también habían «optado» por Alemania, contaban con que Maria C. todavía vivía, como muestra la misiva que enviaron entonces tras instalarse en su nueva patria. La médica encargada les transmitió en su respuesta el fallecimiento de la paciente.

Los informes de Zwiefalten también recogen algún caso de suicidio entre los pacientes: «Un enfermo que había llegado aquí el 26 de mayo de 1940 procedente del Tirol del Sur (esquizofrenia) fue encontrado ahorcado a las 4 de la mañana en su dormitorio, que compartía con otros 5 enfermos. Se había colgado de un tubo de conducción de vapor con una tira de tela arrancada de una sábana. Los intentos por reanimarlo fueron infructuosos. El cadáver fue levantado al día siguiente por el tribunal. No hubo constancia de ningún comportamiento punible por parte del personal sanitario, toda vez que el personal no conocía que el enfermo tenía «tendencias suicidas» ${ }^{26}$. El informe médico anual del centro de Zwiefalten informa objetiva y sobriamente del suicidio de este paciente. Pero nada se dice sobre la identidad de este hombre suicidado justo cuando acababa de retornar al Reich, supuestamente «a su casa».

\section{UN CAMINO DE VUELTA: LA HISTORIA DE LA POSGUERRA}

La salida de los surtiroleses de los psiquiátricos de Württemberg fue algo complicado. En enero de 1941, cuatro pacientes psiquiátricos surtiroleses, por deseos de sus familiares, fueron trasladados de vuelta de Württemberg a Pergine por orden del DUS. Pero dicho traslado fue excepcional. Sabemos que sólo 38 pacientes surtiroleses del conjunto de los tres centros de Württemberg fueron egresados antes de 1945 y enviados con sus familiares asentados en el Reich ${ }^{27}$.

Ya acabada la guerra, otra paciente, Hilde P., consiguió también salir de Zwiefalten, aunque no sin dificultades $^{28}$. Nacida en 1915 , esta mujer soltera trabajaba como «muchacha doméstica». Llegó a Zwiefalten con una esquizofrenia en un estadio avanzado. A partir de 1947, con la mejora de su estado, pidió repetidamente ser dada de alta. Al realizarse, en 1948, un recuento de los surtiroleses susceptibles para un posible retorno, se manifestó a favor de permanecer en Alemania. Pero un año más tarde cambió de opinión y contactó con su hermano y con una tía para volver a su patria. Ambos le dieron una respuesta negativa. El hermano escribió a la dirección del centro: «No conozco otro lugar en el que estuviera mejor atendida que con ustedes». Finalmente, Hilde P. respondió a una oferta de trabajo publicada en la prensa como «sirvienta o ayudante de cocina» en la Clínica Neurológica Universitaria de Tübingen. Al no ser aceptada, solicitó, esta vez con éxito, un puesto de trabajo en la casa de un panadero con tres niños en Cannstatt. El 6 de agosto de 1949 llegó una carta a la dirección de la clínica comunicando que Hilde P. había aparecido sorprendentemente en casa de su tía en Innsbruck. El 7 de octubre de 1949 Hilde P. se disculpó por escrito con su antigua empleadora por haberse marchado sin permiso: «Deseaba a toda costa recuperar totalmente mi libertad», dijo justificando su huida.

Hasta 1945 sólo abandonaron Zwiefalten 16 pacientes en total; la mayor parte de ellos fueron recogidos por sus familiares o egresados a consecuencia de su insistencia.

Cuando a finales de los años cuarenta y principios de los cincuenta se abrió por razones políticas la posibilidad de repatriar a los pacientes procedentes de Italia, los internados en los psiquiátricos de Württemberg no pudieron acogerse a ella. Según el contrato de "opción» ya no eran ciudadanos italianos y, como tampoco habían obtenido la ciudadanía alemana, fueron tratados como apátridas y quedó vetado su regreso (Kanis-Seyfried, 2014, p. 504).

Sólo a partir de 1982 pudieron ser devueltos a sus familias ocho pacientes y otros dos obtuvieron un permiso de estancia permanente en residencias de ancianos del Tirol del Sur. Esta modesta repatriación se debió a un original proyecto de finales del verano de 1974 en el psiquiátrico de Schussenried. Para hacer posible que los 37 surtiroleses todavía supervivientes hiciesen al menos una visita a su patria, se les ofreció la posibilidad de realizar un viaje vacacional de varios días, asistidos por personal sanitario y por un médico, al Tirol del Sur. Como la mayoría, de edad avanzada, no tenían ya ningún contacto familiar, se intentó previamente por el centro localizar posibles familiares. Estos viajes tuvieron una excelente acogida hasta que fueron suspendidos en 1988 por la avanzada edad de los surtiroleses supervivientes, ya que en repetidas ocasiones se consiguió contactar con familiares y hacer posible un reencuentro ${ }^{29}$.

Josef D. fue el último paciente surtirolés en regresar a casa en el año 2002. Cuando murió, en septiembre de 1998, fue inhumado en Zwiefalten. Su deseo de recibir sepultura en Sankt Ulrich, su lugar natal en Val Gardena, fracasó en un principio por trabas burocráticas, pero finalmente fue enterrado allí cuatro años después (Müller y Kanis-Seyfried, 2013, pp. 37-43; Kanis-Seyfried, 2014, p. 509). 


\section{EL CAMBIO DE FUNCIÓN DE LOS ESPACIOS PSIQUIÁ- TRICOS: REFLEXIÓN Y BALANCE}

Los relatos aquí presentados ofrecen una imagen muy dispar de las funciones de las instituciones psiquiátricas. El psiquiátrico de Zwiefalten sirvió desde 1940 como centro de paso para pacientes posteriormente asesinados en Grafeneck dentro de la eutanasia centralizada. Zwiefalten tuvo también casos de eutanasia descentralizada confirmados por sentencia judicial, entre ellos los efectuados bajo la responsabilidad de la doctora Martha Fauser, posteriores a la finalización allí de la eutanasia centralizada. Se calcula que en esta institución perdieron la vida más de 1.000 pacientes por estos procedimientos atentatorios de la dignidad humana. Tras el final de la guerra, las dependencias de Zwiefalten sirvieron de clínica de restablecimiento para niños, tanto de la propia ciudad como reubicados.

El sanatorio de Weissenau vio transformados en hospital militar una importante parte de sus edificios debido a los planes de guerra. Igual sucedió con el psiquiátrico de Reichenau, situado al suroeste de Weissenau, junto a Constanza. Parte de los edificios de Weissenau sirvieron también para alojar a los extranjeros forzados a trabajar en la industria armamentística de la vecina Friedrichshafen. Weissenau alojó también a los enfermos psíquicos que había entre ellos. De estas dos instituciones, Weissenau y Reichenau, unas 700 personas de cada una fueron deportadas a Grafeneck y asesinadas allí. El centro de Schussenried no se vio afectado por las planificaciones militares. La tasa de mortalidad fue elevada también en esta institución después del fin de la eutanasia centralizada, si bien nunca alcanzó los niveles de Zwiefalten.

La lista de estos cambios forzosos en la finalidad de los centros podría seguir ampliándose, pero en esta contribución nos limitaremos a mencionar otros dos casos especialmente interesantes y estrechamente vinculados al tratado de "opción» suscrito entre Alemania e Italia ${ }^{30}$.

El primero corresponde al sanatorio de Illenau, en Baden, junto a Achern, en donde, según parece, ya en 1939 su director médico, Hans Römer, recibió la orden de desalojo y del consiguiente traslado de pacientes. No está claro en qué medida los promotores de la orden tenían constancia de la campaña occidental (el ataque a Francia) planeada por la Wehrmacht. Römer parece que no dio el consentimiento para el desmantelamiento de su hospital (de hecho planeaba la creación de una «Clínica Neurológica de Baden Central» de mayores dimensiones), ni tampoco para el traslado de pacientes, hasta la primavera de 1940. Römer intentó así, presumiblemente, retrasar dicho proceso. Sin embargo, hoy sabemos que las primeras 75 personas deportadas desde Illenau el 18 de junio de 1940 encontraron la muerte en Grafeneck dentro de la eutanasia centralizada. Por iniciativa de Römer, parte de los pacientes de Illenau fueron dados de alta y parte trasladados al centro de Emmendingen. Pero las restantes personas encontraron la muerte en Grafeneck. El centro existió como sanatorio psiquiátrico sólo hasta el 19 de diciembre de 1940. A partir de 1941, y de forma similar a lo que ocurrió con Reichenau, Illenau fue reconvertido en un Centro de Educación Político Nacional (Nationalpolitische Erziehungsanstalt) o, en sus siglas oficiales, NPEA, si bien coloquialmente se usó más la abreviatura NAPOLA, por Nationalpolitische Lehranstalt, (es decir, Centro de Enseñanza Político Nacional). Concebidas en un primer momento como escuelas para la formación de élites nacionalsocialistas, estas instituciones estuvieron sometidas desde 1936 a las SS. Hasta el final de la guerra se fundaron 43 NAPOLAs en el Reich, tres de ellas femeninas.

Otra función que se dio a las edificios de Illenau $-\mathrm{y}$ la de más interés aquí- fue la de Escuela del Reich para Alemanes Étnicos (Reichschule für Volksdeutsche). Aquí vivieron durante más de cuatro años entre cuatrocientas y quinientas chicas surtirolesas llegadas a Baden por el Acuerdo para la Opción. Como en la Italia fascista la enseñanza en lengua alemana estaba prohibida bajo multas severas, para aprender a escribir correctamente en su lengua materna alemana estas muchachas tuvieron que asistir a escuelas muy apartadas de sus domicilios familiares ${ }^{31}$.

La nueva función asignada a Illenau como Centro de Enseñanza Político Nacional inicialmente sólo femenino fue modificada en 1943/44. Desde entonces, se llevó a cabo allí también para muchachos la formación ideológica y la "germanización» cultural. Posiblemente esto se debió el desmantelamiento del centro alsaciano de Rufach, en el que previamente habían sido acuartelados entre 600 y 650 varones menores de edad procedentes del Tirol del Sur cuyos padres habían votado a favor del Reich. En Rufach, al sur de Colmar, la Wehrmacht había expulsado, tras la ocupación de estos territorios franceses, a los pacientes del sanatorio, así como a sus médicos y demás personal sanitario, al menos a los que no habían huido al interior de Francia nada más empezar las hostilidades. A continuación se había establecido allí una NAPOLA para muchachos alemanes étnicos, así como una escuela con enseñanza primaria, media y superior. 
Los chicos alojados primero en Rufach y las chicas acomodadas en Illenau sumaron un total de más de mil menores cuya inmigración a Baden y a la Alsacia ocupada desde Tirol del Sur fue voluntaria. Las personas oriundas del Tirol del Sur y de los territorios colindantes habían recibido la promesa de que podrían asentarse o bien en el denominado Altreich (Antiguo Reich) o bien en los territorios rápidamente conquistados, y que allí encontrarían una nueva patria y un pasaporte alemán en vez de italiano.

Estas oleadas de inmigración en consonancia con la denominada biopolítica del nacionalsocialismo deben ser claramente diferenciadas, por el hecho de que fueron voluntarias (es decir, deseadas por los sujetos), del traslado arriba descrito de personas enfermas y ancianas desde Italia hacia Württemberg. La reubicación de la población sana del Tirol del Sur en Baden y en Alsacia correspondió a una acogida política bajo el lema, entonces muy extendido y que suscitaba simpa-

\section{NOTAS}

1. Sobre los pacientes de instituciones psiquiátricas del Tirol del Sur, pueden consultarse entre otros: Pantozzi, 1989; Hinterhuber, 1995; y May, 1996.

2. Hay que mencionar aquí las investigaciones en curso de la Universidad de Innsbruck, del Südtiroler Landesarchiv y del Hospital Psiquiátrico de Hall i. T. Durante unas obras se halló en el terreno del actual hospital una fosa común. La identificación de los cuerpos allí enterrados ha sido en un primer momento dudosa.

3. Apunte del expediente Luig (ADERSt Bolzano) relativo la salida de los enfermos mentales del 25/4/1940, BA Berlin, R 49/2146, sin paginar. Véase también: Fiebrandt; Rüdenburg; Müller, 2012a, p. 165.

4. Informe sobre el transporte de optantes alemanes étnicos con enfermedades mentales de Pergine a Zwiefalten (Württemberg) del 18/6/1940, Dr. Simek, BA Berlin, R $49 / 2265$, p. 2. Véase también: Fiebrandt; Rüdenburg; Müller, 2012a, pp. 165 y ss.

5. En algunos pocos casos el derecho a optar fue ejercido - sin que hubiese un acuerdo jurídicamente válido- por los parientes, pero en la mayoría de los casos lo ejerció la administración de la provincia de Bolzano. Véase: Fiebrandt; Rüdenburg; Müller, 2012a, pp. 166 y ss.

6. Informe del doctor Stegmann sobre la recepción de enfermos mentales de etnia alemana procedentes de sanatorios del norte de Italia del 29/5/1940, dirigido al Ministerio del Interior de Württemberg en Stuttgart, Archivo del Zentrum tía, de «Heim ins Reich!» (algo así como « ¡De vuelta a casa, de vuelta al Reich!»). Los traslados que afectaron a las personas enfermas sucedieron, como se ha descrito, contra la voluntad de parte de los transferidos, y a veces incluso sin el conocimiento de sus familiares. Algunas de estas personas eran solamente ancianos sin contacto previo alguno con la terapia psiquiátrica. Es decir, que habían sido "psiquiatrizadas» a mala fe (para favorecer su traslado) por personal médico italiano ${ }^{32}$. En el Reich alemán, y a diferencia de los surtiroleses sanos y aptos para el trabajo, estos no fueron naturalizados. A partir de este hecho puede deducirse el escaso «valor vital» (Lebenswert) que los responsables nacionalsocialistas atribuyeron a estas personas.

\section{AGRADECIMIENTOS}

Trabajo realizado en el marco del proyecto de investigación HAR2012-37754-C02-02 (Ministerio de Economía y Competitividad - España).

für Psychiatrie (ZfP) Südwürttemberg, sede Münsterklinik Zwiefalten, carpeta Surtiroleses. Véase también: KanisSeyfried, 2014, p. 499.

7. Informe anual del sanatorio de Weissenau 1940 del 2/5/1941 (Dr. Bischoff), Archivo del ZfP Südwürttemberg, sede Ravensburg-Weissenau.

8. Carta del doctor Stegmann, sanatorio de Zwiefalten, Württemberg, al Ministro del Interior, $n$ ㅇ 266, 29 de mayo de 1940, legado del doctor Johannes May, ZfP Südwürttemberg, Archivo de Zwiefalten. Véase Kanis-Seyfried, 2014, p. 499.

9. Carta del doctor Stegmann, sanatorio de Zwiefalten, Württemberg, al señor Ministro del Interior, no 266, 29 de mayo de 1940, legado del doctor Johannes May, ZfP Südwürttemberg, Archivo de Zwiefalten.

10. Declaración de la doctora Helene Volk, Proceso Grafeneck Tübingen 1949, Staatsarchiv Sigmaringen, Fondo Wü 29/3T1. Véase: Kanis-Seyfried, 2014, pp. 500 y ss. Puede consultare aquí también: Kinzig; Stöckle, 2011; así como Müller; Kinzig y Stöckle, 2010; Pollmann y Müller, 2012.

11. Escrito no 661, sanatorio de Schussenried, Württemberg, dirigido al Ministerio del Interior de Stuttgart, a la atención del doctor Stähle, 5 de noviembre de 1940, legado del doctor Johannes May, ZfP Südwürttemberg, Archivo de Zwiefalten. Véase también: Kanis-Seyfried, 2014, p. 501.

12. Carta del consejero médico jefe Mauthe al Ministro del Interior, 9 de diciembre de 1949, legado del doctor Johannes 
May, ZfP Südwürttemberg, Archivo de Zwiefalten. El doctor Otto Mauthe era, en tanto oficial encargado en el Ministerio del Interior de Württemberg, el responsable del sistema psiquiátrico y en 1949 tuvo que responder durante el Proceso Grafeneck por el asesinato de enfermos procedentes de centros de Württemberg.

13. Copia de la carta de queja de los pacientes surtiroleses de Schussenried 1941, legado del doctor Johannes May, ZfP Südwürttemberg, Archivo de Zwiefalten. Esta cita pone de manifiesto nítidamente que la crítica sobre las inadecuadas condiciones de reubicación no implicó una actitud crítica contra el sistema por parte de los afectados. Véase también: Kanis-Seyfried, 2014, p. 502.

14. Carta del doctor Stähle al Departamento de Exteriores de la Cámara Médica del Reich de Berlín, 19 de agosto de 1941, legado del doctor Johannes May, ZfP Südwürttemberg, Archivo de Zwiefalten.

15. Carta del doctor Stähle al Departamento de Exteriores de la Cámara Médica del Reich en Berlín, 19 de agosto de 1941, legado del doctor Johannes May, ZfP Südwürttemberg, Archivo de Zwiefalten. Véase también: Kanis-Seyfried, 2014, pp. 497-502.

16. Informe anual del sanatorio de Weissenau 1940 del 5/3/1942 (Dr. Bischoff), ZfP Südwürttemberg, sede Ravensburg-Weissenau, véase aquí el punto 1: Allgemeine Krankenbewegung (Movilización general de los enfermos).

17. Sobre los homicidios inducidos por medicamentos, también objeto del Proceso Grafeneck de 1949, véase: Kinzig; Stöckle, 2011.

18. Véase a este respecto la historia clínica de la paciente Emilia V., que fue archivada en el Staatsarchiv (StA) Sigmaringen ( $n$ o de historia Wü 68/1 Acc. 4/1992 3832/5831). Para más detalles, véase: Fiebrandt; Rüdenburg; Müller, 2012a, pp. 185-187.

19. Historia clínica del paciente Walter D., Staatsarchiv (StA) Sigmaringen. Véase también Kanis-Seyfried, 2014, pp. 491-514.

20. Esta historia fue archivada en el actual Staatsarchiv (StA) Sigmaringen (no de historia Wü 68/1 Acc. 4/1992 3832/5831).

21. Formulario de registro de la paciente Emilia V. del 21/2/1941, StA Sigmaringen, Wü 68/1 Acc. 4/1992, 3832/5831. Estos datos están confirmados por sus familiares. Agradecemos a Carla Giacomozzi, de Bolzano, estas informaciones.

22. Historia clínica de la paciente Emilia V., StA Sigmaringen, Wü 68/1 Acc. 4/1992, 3832/5831.

23. Del Centro de Schussenried al DUS, 20/6/1941, StA Sigmaringen, Wü 68/1 Acc. 4/1992, 3832/5831. En un intercambio de correspondencia de julio de 1943, ya después de la muerte de la paciente, se habla además de un paquete que un familiar echaba en falta.

24. Historia clínica de Emilia V., StA Sigmaringen, Wü 68/1 Acc. 4/1992, 3832/5831, bajo el epígrafe 'Terapia'.

25. De Schussenried al DUS, 29/6/1943, StA Sigmaringen, Wü 68/1 Acc. 4/1992, 3832/5831.

26. Informe anual de Zwiefalten 1940, ZfP Südwürttemberg, Archivo de Zwiefalten, p. 9. Véase también: Kanis-Seyfried, 2014, pp. 492 y ss.

27. Véase aquí y en lo que sigue a continuación: Apuntes manuscritos, May, Johannes, carpeta Tiroleses del Sur de Schussenried (sin paginar), legado del doctor Johannes May, ZfP Südwürttemberg, Archivo de Zwiefalten. Véase también: May, 1995.

28. Historia clínica de Hilde P., legado del doctor Johannes May, ZfP Südwürttemberg, Archivo de Zwiefalten. Véase también Kanis-Seyfried, 2014, p. 507.

29. Legado del doctor Johannes May, ZfP Südwürttemberg, Archivo de Zwiefalten.

30. Sobre el spatial turn en relación con la historia de la medicina, véase entre otros: Topp; Moran y Andrews, 2007.

31. Al principio se hizo cargo de ellas un párroco católico de Oberachern y, siguiendo estrictamente los planes de estudio alemanes, fueron educadas después en una escuela primaria y media, en una escuela superior femenina y en una escuela superior de comercio.

32. Para el análisis detallado de la política estatal de reubicación en el marco del Acuerdo para la Opción, véase también: Fiebrandt; Rüdenburg y Müller, 2012b, Respecto a su comparación con otras reubicaciones, véase: Fiebrandt, 2014; respecto al Tirol del Sur, véase: Wedekind, 2009. 


\section{BIBLIOGRAFÍA}

Arreghini, Ermanno (1995), “Option: eine freie Entscheidung oder Abschiebung von Unzurechnungsfähigen?". En: Verband Angehöriger und Freunde psychisch Kranker: Wahnsinn und ethnische Säuberung. Deportation und Vernichtung psychisch Kranker aus Südtirol 1939-45, Bozen, pp. 37-41.

Faulstich, Heinz (1998), Hungersterben in der Psychiatrie 19141949, Freiburg, Lambertus, pp. 301-305, pp. 351-355.

Fiebrandt, Maria; Rüdenburg, Bodo y Müller, Thomas (2012a), "Nationalsozialistische Erbgesundheitspolitik und Psychiatrie. Die 'Umsiedlung' Südtiroler Psychiatriepatienten nach Württemberg im Rahmen des deutsch-italienischen Optionsvertrages ab 1939". En: Müller, Thomas; Reichelt, Bernd y Kanis-Seyfried, Uta (eds.), Nach dem Tollhaus. Zur Geschichte der KöniglichWürttembergischen Staatsirrenanstalt Zwiefalten, Zwiefalten, Psychiatrie und Geschichte, pp. 154-190.

Fiebrandt, Maria; Rüdenburg, Bodo y Müller, Thomas (2012b), “Von Südtirol nach Württemberg. Die 'Umsiedlung' Südtiroler Psychiatriepatienten im Rahmen des deutsch-italienischen Optionsvertrages ab 1939", Gesnerus. Schweizerische Zeitschrift für die Geschichte der Medizin und der Naturwissenschaften, 69(2), pp. 297-329.

Fiebrandt, Maria (2014), Auslese für die Siedlergesellschaft. Die Einbeziehung Volksdeutscher in die NS-Erbgesundheitspolitik im Kontext der Umsiedlungen 1939-1945, Göttingen, Vandenhoeck \& Ruprecht.

Helmut, Alexander (1993), “Die Umsiedlung der Südtiroler 1939-1945". En: Helmut, Alexander; Lechner, Stefan y Leidlmair, Adolf (eds.), Heimatlos. Die Umsiedlung der Südtiroler, Wien, Tiroler Landesinstitut, pp. 43-179.

Hinterhuber, Hartmann (1995), Ermordet und vergessen. Nationalsozialistische Verbrechen an psychisch Kranken und Behinderten in Nord- und Südtirol, Innsbruck, Integrative Psychiatrie.

Kanis-Seyfried, Uta (2014), “Die Sehnsucht nach der Heimat bleibt. Die Folgen der NS-Bevölkerungspolitik am Beispiel der Psychiatriepatienten aus Südtirol". En: Karenberg, Axel y Kumbier, Ekkehardt (eds.), Schriftenreihe der Deutschen Gesellschaft für die Geschichte der Nervenheilkunde, Würzburg, Königshausen und Neumann, pp. 491-514.

Kinzig, Jochen y Stöckle, Thomas (2011) (eds), 60 Jahre Tübinger Grafeneck-Prozess. Betrachtungen aus historischer, juristischer, medizinethischer und publizistischer Perspektive, Zwiefalten, Psychiatrie und Geschichte.

Kretschmer, Manfred (1983), "Von der Staatsirrenanstalt zum Akademischen Krankenhaus. Die Heilanstalt im Hitler-Reich".
En: Eitel, Peter (ed.), Weissenau. Geschichte und Gegenwart, Sigmaringen, Jan Thorbecke, pp. 344-347.

May, Johannes (1995), "Südtiroler Patienten in Baden-Württemberg. Versuche ihrer Repatriierung", Verband Angehöriger und Freunde psychisch Kranker: Wahnsinn und ethnische Säuberung. Deportation und Vernichtung psychisch Kranker aus Südtirol 1939-45, Bozen, pp. 55-64.

May, Johannes (1996) "Die Staatliche Heilanstalt Schussenried in den Jahren 1933 bis 1945", En: Pretsch, H. J. (ed.), Euthanasie. Krankenmorde in Südwestdeutschland, Zwiefalten, Geschichte und Psychiatrie, pp. 74-83.

Müller, Thomas; Kinzig, Jörg y Stöckle, Thomas (2010) (eds.), "Die deutsche Psychiatrie, der Patientenmord im Nationalsozialismus, die juristischen Folgen und die Erinnerungspolitik". En: Türkisches Jahrbuch für Studien zu Ethik und Recht in der Medizin 2/3, pp. 29-60.

Müller, Thomas y Kanis-Seyfried, Uta (2013), “Die Südtiroler Patienten in Württemberg. Nationalsozialistische und italienische Bevölkerungspolitik nach 1939", Schwäbische Heimat 64, 1, pp. 37-43.

Pantozzi, Giuseppe (1989), Die brennende Frage. Geschichte der Psychiatrie in den Gebieten von Bozen und Trient, 18301942, Autonome Provinz Bozen - Südtirol, Bozen.

Pollmann, Iris y Müller, Thomas (2012), “Die württembergische Psychiaterin Martha Fauser". En: Müller, Thomas; Reichelt, Bernd y Kanis-Seyfried, Uta (eds.), Nach dem Tollhaus. Zur Geschichte der Königlich-Württembergischen Staatsirrenanstalt Zwiefalten, Zwiefalten, Psychiatrie und Geschichte, pp. 116-128.

Rüdenburg, Bodo (1996), “Die 'Aktion T4' in Zwiefalten”. En: Pretsch, H. J. (ed.), Euthanasie. Krankenmorde in Südwestdeutschland, Zwiefalten, Geschichte und Psychiatrie, pp. 37-46.

Süß, Winfried (2003), Der Volkskörper im Krieg. Gesundheitspolitik, medizinische Versorgung und Krankenmord im nationalsozialistischen Deutschland, 1939-1945, München, Oldenburg.

Stuhlpfarrer, Karl (1985), Umsiedlung Südtirol 1939-40, Wien, Löcker.

Topp, Leslie; Moran, James y Andrews, Jonathan (eds.) (2007), Madness, Architecture and the Built Environment: Psychiatric Spaces in Historical Contex, London, Taylor Francis Ltd.

Wedekind, Michael (2009), "Planung und Gewalt: Raumordnung und Bevölkerungsplanung im Kontext der Umsiedlung Tirol", Geschichte und Region/Storia e regione 18(2), pp. 71-109. 